\title{
Topological pumping of quantum correlations
}

\author{
T. Haug, ${ }^{1,2, *}$ L. Amico, $, 2,3,4,5,6$ L.-C. Kwek,,${ }^{2,5,7,8}$ W. J. Munro, ${ }^{1}$ and V. M. Bastidas $\oplus^{1, \dagger}$ \\ ${ }^{1}$ NTT Basic Research Laboratories and Research Center for Theoretical Quantum Physics, \\ 3-1 Morinosato-Wakamiya, Atsugi, Kanagawa 243-0198, Japan \\ ${ }^{2}$ Centre for Quantum Technologies, National University of Singapore, 3 Science Drive 2, Singapore 117543, Singapore \\ ${ }^{3}$ Consiglio Nazionale delle Ricerche-MATIS-IMM and Dipartimento di Fisica e Astronomia, Universitá Cnia, \\ Via S. Soa 64, 95127 Catania, Italy \\ ${ }^{4}$ Istituto Nazionale di Fisica Nucleare Laboratori Nazionali del Sud, Via Santa Sofia 62, I-95123 Catania, Italy \\ ${ }^{5}$ MajuLab, Centre National de la Recherche Scientifique-UNS-NUS-NTU International Joint Research Unit, UMI 3654, Singapore \\ ${ }^{6}$ LANEF “Chaire d'excellence," Universitè Grenoble-Alpes and Centre National de la Recherche Scientifique, F-38000 Grenoble, France \\ ${ }^{7}$ Institute of Advanced Studies, Nanyang Technological University, 60 Nanyang View, Singapore 639673, Singapore \\ ${ }^{8}$ National Institute of Education, Nanyang Technological University, 1 Nanyang Walk, Singapore 637616, Singapore
}

(Received 20 May 2019; revised manuscript received 2 October 2019; accepted 3 January 2020; published 7 February 2020)

\begin{abstract}
Topological pumping and duality transformations are paradigmatic concepts in condensed matter and statistical mechanics. In this paper, we extend the concept of topological pumping of particles to topological pumping of quantum correlations. We propose a scheme to find pumping protocols for highly correlated states by mapping them to uncorrelated ones. We show that one way to achieve this is to use dualities, because they are nonlocal transformations that preserve the topological properties of the system. By using them, we demonstrate that topological pumping of kinks and clusterlike excitations can be realized. We find that the entanglement of these highly correlated excitations is strongly modified during the pumping process and the interactions enhance the robustness against disorder. Our paper paves the way to explore topological pumping beyond the notion of particles and opens an avenue to investigate the relation between correlations and topology.
\end{abstract}

DOI: 10.1103/PhysRevResearch.2.013135

\section{INTRODUCTION}

In 1983, Thouless demonstrated that the topological properties of the wave function of an extended system can be exploited to realize quantum pumps (see [1-7]). This mechanism can perform topologically protected quantum transport, which is robust against disorder and weak interactions [2]. The remarkable progress of quantum technology has allowed the implementation of Thouless's idea to unprecedented degrees. Recently, quantum pumping of particles has been realized in diverse platforms ranging from ultracold optical superlattices [8,9] to waveguide arrays [10,11]. In the context of quantum simulation, quantum pumping in low-dimensional systems can be used to simulate higher dimensional quantum systems [12]. For example, recent experiments have demonstrated that topological pumping in two-dimensional systems can be used to explore the exotic physics of quantum Hall effect in four dimensions using cold atoms [13] and photonic systems [14].

\footnotetext{
*tobias.haug@u.nus.edu

†victor.bastidas@lab.ntt.co.jp
}

Published by the American Physical Society under the terms of the Creative Commons Attribution 4.0 International license. Further distribution of this work must maintain attribution to the author(s) and the published article's title, journal citation, and DOI.
In this paper, we address the problem of topological pumping of quantum correlations. The cornerstone of our approach is to map the highly correlated states that we want to pump to uncorrelated ones, for which topological pumping protocols are well known [see Fig. 1(a)]. Thus, the topological pumping of correlated states can be obtained by a suitable inverse mapping. However, in general, finding the right mapping is a nontrivial problem, since it has to reduce the correlations of the states, while preserving the topological properties of the system. Here, we show that dualities can be exploited to achieve this goal [15-24]. While the duality can change the entanglement of the collective excitations [24,25], the topological properties of the energy bands are unaltered. In this way, we can extend the idea of topological pumping to transport highly entangled excitations. Due to its topological nature, the proposed quantum pumping is protected against disorder and some type of interactions.

To illustrate our approach, we show how to perform topological pumping of cluster and kink excitations [26,27], which are related to spin flips by duality, as depicted in Fig. 1(b). We are now able to explore the pumping dynamics of these entangled excitations for the first time. We show that bipartite entanglement is dramatically affected by duality and pumping: spin flips become highly entangled at the anticrossings of the spectrum. Contrary to this, the entanglement present in kinks and cluster states is reduced or stays constant for specific bipartite divisions. One of the most appealing features of topological pumping is its robustness against disorder. In most cases, introducing interactions between excitations 
(a)

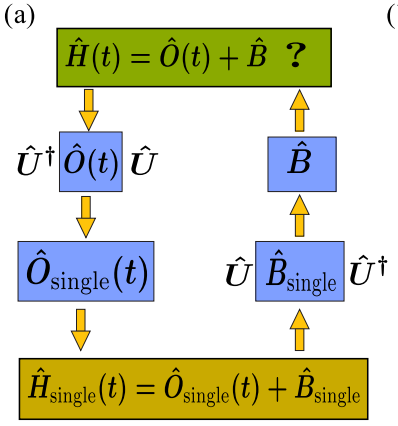

(b)

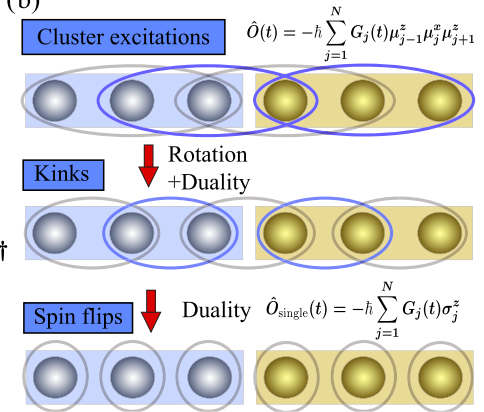

FIG. 1. Topological pumping of highly correlated states. (a) Depiction of our scheme, which allows us to pump correlations of the operator $\hat{O}(t)$. The main challenge is to find an operator $\hat{B}$ that enables the pumping protocol. To do this, one needs to find a unitary transformation $\hat{U}$ that maps the many-body operator $\hat{O}(t)$ to a single-particle operator $\hat{O}_{\text {single }}(t)$. In the single-particle picture we know what operator $\hat{B}_{\text {single }}$ is required in order to pump particles. By transforming the operator back, we can construct the operator $\hat{B}$. (b) A particular example where duality transformations enable us to develop a protocol to pump cluster states. Under duality, the cluster excitations map to kinks and finally to spin-flip excitations, which can be mapped to single particles for which pumping is known.

destroys topological transport [28]. However, we find that for a particular type of interaction topological pumping is still possible and, furthermore, robustness against certain types of disorder is strongly enhanced.

\section{TOPOLOGICAL PUMPING AND HIGHLY CORRELATED STATES}

In the following, we discuss our general procedure, which is schematically presented in Fig. 1(a). We consider a general Hamiltonian of the form

$$
\hat{H}(t)=\hat{O}(t)+\hat{B},
$$

where the operator $\hat{O}(t)$ creates many-body correlations in the system. Our goal is to pump these correlations. In principle, the task can be achieved by finding a time-independent operator $\hat{B}$ that is noncommuting with $\hat{O}(t)$. However, to find the right operator can be nontrivial, because, in general, not all operators $\hat{B}$ allow topological pumping. The operator $\hat{B}$ must couple the eigenstates of $\hat{O}(t)$ to lift the degeneracies between them at all times $t$ during the pumping process. In this paper, we aim to find a unitary transformation $\hat{U}$ that allows us to map the correlated problem to a simpler one, for which the topological pumping protocol is well known. The unitary operator $\hat{U}$ has to be sufficiently nonlocal in space to uncorrelate $\hat{O}(t)$. If such unitary mapping can be obtained, then $\hat{O}(t)$ can be transformed to a single-particle operator $\hat{O}_{\text {single }}(t)$. Then, the corresponding single-particle pumping operator $\hat{B}_{\text {single }}$ can be found by resorting to known frameworks of topological pumping of single particles:

$$
\hat{H}_{\text {single }}(t)=\hat{O}_{\text {single }}(t)+\hat{B}_{\text {single }} .
$$

Finally, the correlation pumping operator $\hat{B}$ is obtained via the inverse transformation $\hat{B}=\hat{U} \hat{B}_{\text {single }} \hat{U}^{\dagger}$. We discuss below an example of our general scheme, where $\hat{U}$ is constructed by

combining duality and rotations in a spin system. In order to highlight the capability of our scheme, we show below how to perform topological pumping of cluster and kink states.

\section{A. Mapping cluster states to spin flips}

Let us begin by considering the time-dependent operator $\hat{O}(t)=-\hbar \sum_{j=1}^{N} G_{j}(t) \mu_{j-1}^{z} \mu_{j}^{x} \mu_{j+1}^{z}$, where $\mu_{j}^{\alpha}(\alpha \in\{x, y, z\})$ are Pauli matrices acting on the $j$ th site. The eigenstates of this operator are clusterlike states, which are highly entangled objects relevant for quantum computation [29] or quantum memories [30]. In order to be able to pump cluster states, we need to find a suitable unitary operator $\hat{U}$. With this aim, let us consider the Kramers-Wannier duality $\tau_{j}^{z} \tau_{j+1}^{z}=\mu_{j}^{x}$, $\tau_{j}^{x}=\mu_{j}^{z} \mu_{j+1}^{z}, \tau_{j}^{y} \tau_{j+1}^{y}=\mu_{j}^{z} \mu_{j+1}^{x} \mu_{j+1}^{z}$. Here, $\tau_{j}^{\alpha}$ are the Pauli matrices after the dual transformation $[15,16]$. By applying the duality transformation, rotating the spins around the $x$ axis, and then applying the duality again [see Fig. 1(b)], one can remove the correlations in $\hat{O}(t)$. This allows us to map the latter to a single-particle operator $\hat{O}_{\text {single }}(t)=-\hbar \sum_{j=1}^{N} G_{j}(t) \sigma_{j}^{x}$ in terms of new Pauli matrices $\sigma_{j}^{x}$. In contrast to the original operator $\hat{O}(t)$, the eigenstates $\hat{O}_{\text {single }}(t)$ are uncorrelated states known as spin flips. Now it is quite direct to find an operator $\hat{B}_{\text {single }}$ enabling us to perform topological pumping of spin flips. To do this, let us consider the one-dimensional quantum Ising model in a transverse field [26]

$$
\hat{H}^{\mathrm{flip}}(t)=\hbar \sum_{j=1}^{N}\left[-G_{j}(t) \sigma_{j}^{x}+J \sigma_{j}^{z} \sigma_{j+1}^{z}\right],
$$

which is a paradigmatic model in condensed matter and statistical physics. We consider a transverse field strength $G_{j}(t)=g_{0}+g_{1} \cos \left[2 \pi(j-1) b+\omega t+\phi_{0}\right]$, which is adiabatically modulated in time. Here $\omega$ is the frequency of the drive and its period is $T=2 \pi / \omega$. The parameters $\phi_{0}$ and $1 / b$ determine the initial phase shift and the spatial period of the modulation, respectively. In addition, $J$ is the strength of the spin-spin interaction. We assume periodic boundary conditions $\sigma_{j}^{\alpha}=\sigma_{j+N}^{\alpha}$ and explore the specific case $b=1 / 3$. However, our results remain valid for any rational value $b=$ $p / q$, where $p$ and $q$ are coprimes. In the following, we will show that $\hat{B}_{\text {single }}=\hbar J \sum_{j=1}^{N} \sigma_{j}^{z} \sigma_{j+1}^{z}$ is the operator that allows us to perform topological pumping of spin flips.

\section{B. Topological pumping of cluster states}

Due to the spatial modulation of the transverse field, the Ising Hamiltonian of Eq. (3) exhibits topological features that we can exploit to perform topological pumping. To gain some intuition about this, let us consider the weak-coupling regime $g_{0} \gg J$, where the collective excitations are spin flips. Since the interaction between neighboring spins is small, the total number of excitations $\hat{\mathcal{N}}=1 / 2 \sum_{j=1}^{N}\left(1+\sigma_{j}^{x}\right)$ is approximately conserved. Thus, the spectrum is divided into different bands associated with a fixed number of spin flips (see Fig. 2). Within a given band, the Hamiltonian (3) exhibits the same dynamics as the Aubry-Andre (AA) model [10,31], which is a toy model for topological pumping [1,2]. The current of transported particles is intimately related to the Chern numbers $C$ of the Harper-Hofstadter model [32]. In Appendices A and B 
(a)

(b)

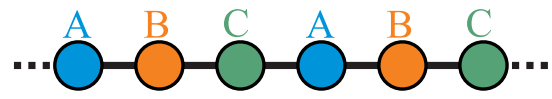

$E / g_{1}$

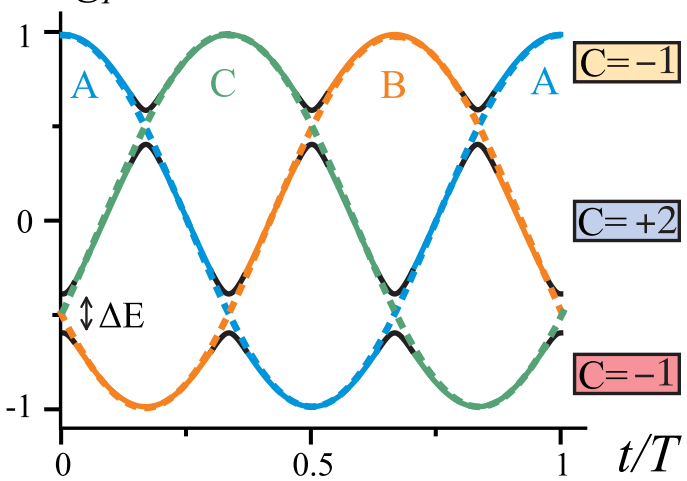

FIG. 2. Topological pumping of spin excitations. (a) Depiction of trimers, where the sites $\mathrm{A}, \mathrm{B}$, and $\mathrm{C}$ have energies $E_{\mathrm{A}}=$ $g_{1} \cos (\omega t), E_{\mathrm{B}}=g_{1} \cos (2 \pi / 3+\omega t)$, and $E_{\mathrm{C}}=g_{1} \cos (4 \pi / 3+\omega t)$, respectively. (b) Instantaneous energies of a single spin-flip excitation per trimer in the limit $g_{0} \gg J$. The dashed lines show the local energies of the sites A (blue), B (orange), and C (green) for $J=0$. The solid lines show the spectrum for $J \neq 0$. At the crossings, the energies of two neighboring spins are degenerate. The spinspin interaction hybridizes the excitations and lifts the degeneracy, creating an energy gap $\Delta E \approx 2 J$. The number of anticrossings is related to the Chern number $C$ of the respective band. For trimers, particles are pumped by $3 C$ sites per driving period.

we describe these models in detail. During the pumping process, the Chern number $C$ determines the number of lattice sites that a single particle is transported per cycle [33]. In other words, the mean position changes as $x(T)-x(0)=$ $1 / 2 \sum_{j=1}^{N} j\left(\left\langle\sigma_{j}^{x}(T)\right\rangle-\left\langle\sigma_{j}^{x}(0)\right\rangle\right)=C$, where $C$ is the Chern number associated to the relevant band, $x(t)=\langle\hat{x}(t)\rangle$, and $\hat{x}=$ $1 / 2 \sum_{j=1}^{N} j\left(\sigma_{j}^{x}+1\right)$ is the position operator. For our choice of $G_{j}(t)$ with a trimer structure, the particle is transported $3 C$ sites per driving period.

From our previous discussion, we conclude that the operator $\hat{B}_{\text {single }}=\hbar J \sum_{j=1}^{N} \sigma_{j}^{z} \sigma_{j+1}^{z}$ allows us to perform topological pumping of single-particle excitations. By applying the inverse of the above transformations, we can construct the operator $\hat{B}=\hbar J \sum_{j=1}^{N} \mu_{j}^{z} \mu_{j+1}^{z}$ that allows us to pump cluster states. This gives rise to the cluster-Ising Hamiltonian [34-37]

$$
\hat{H}^{\text {cluster }}(t)=\hbar \sum_{j=1}^{N}\left[-G_{j}(t) \mu_{j-1}^{z} \mu_{j}^{x} \mu_{j+1}^{z}+J \mu_{j}^{z} \mu_{j+1}^{z}\right] .
$$

By repeatedly applying spin rotations and duality transformations, there is a plethora of complex many-body spin operators that can be reduced to single-particle operators, as we show in Appendix C. These models host excitations with a wide range of topological phases [38], for which we can now construct a pumping protocol. As an example, by using the duality, we can find a Hamiltonian to pump kinks, which are eigenstates of the operator $\hat{O}(t)=-\hbar \sum_{j=1}^{N} G_{j}(t) \tau_{j}^{z} \tau_{j+1}^{z}$. In fact, as the
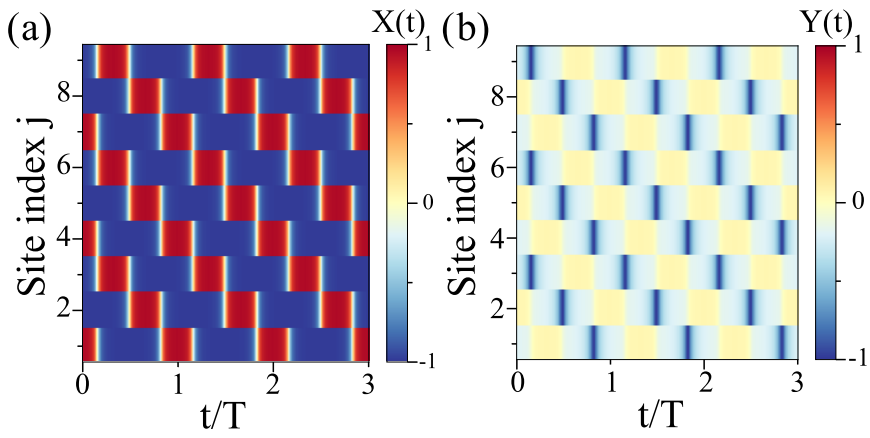

FIG. 3. (a) Evolution of the expectation value of the driving operator of the Hamiltonian. For spin flips [Eq. (3)] $X_{j}(t)=\left\langle\sigma_{j}^{x}\right\rangle$, for the kink model [Eq. (5)] $X_{j}(t)=\left\langle\tau_{j}^{z} \tau_{j+1}^{z}\right\rangle$, and for the cluster-Ising model [Eq. (4)] $X_{j}(t)=\left\langle\mu_{j}^{z} \mu_{j+1}^{x} \mu_{j+2}^{z}\right\rangle$. (b) Expectation value of the nondriven operator of the model Hamiltonians. For spin flips $Y_{j}(t)=$ $\left\langle\sigma_{j}^{z} \sigma_{j+1}^{z}\right\rangle$, for the kink model $Y_{j}(t)=\left\langle\tau_{j}^{x}\right\rangle$, and for the cluster-Ising model $Y_{j}(t)=\left\langle\mu_{j}^{z} \mu_{j+1}^{z}\right\rangle$. The initial state is the eigenstate with one excitation per trimer in the lowest band with Chern number $C=-1$. Increasing or decreasing the number of trimers in the chain does not substantially affect the dynamics. Parameters for all graphs are $N=9, g_{0}=10 J, g_{1}=3 J$, initial phase $\phi_{0}=0$, and frequency $\omega=0.02 J$

Hamiltonian

$$
\hat{H}^{\mathrm{kink}}(t)=\hbar \sum_{j=1}^{N}\left[-G_{j}(t) \tau_{j}^{z} \tau_{j+1}^{z}+J \tau_{j}^{x}\right]
$$

is dual to the Ising Hamiltonian (3), the natural choice of the operator that allows us to pump kinks is $\hat{B}=\hbar J \sum_{j=1}^{N} \tau_{j}^{x}$. During topological pumping, the change of the mean position is related to the Chern number. In the case of Hamiltonian (4), we can show that correlations can be pumped (see Appendix B), as follows:

$$
\begin{aligned}
x(T)-x(0)= & \frac{1}{2} \sum_{j=1}^{N} j\left(\left\langle\mu_{j}^{z} \mu_{j+1}^{x} \mu_{j+2}^{z}(T)\right\rangle\right. \\
& \left.-\left\langle\mu_{j}^{z} \mu_{j+1}^{x} \mu_{j+2}^{z}(0)\right\rangle\right)=C .
\end{aligned}
$$

To illustrate this, we depict the dynamics of topological pumping of quantum correlations in Fig. 3(a).

\section{ENTANGLEMENT DYNAMICS AND TOPOLOGICAL PUMPING}

Kinks and clusters are highly entangled states, whereas spin flips are very close to product states. A natural question that arises is the following: what is the dynamics of entanglement during topological pumping and how does this depend on the character of the excitations? To answer this question we divide the spin chain into two subsystems $\mathrm{A}$ and $\mathrm{B}$; perform a partial trace over $\mathrm{A}, \hat{\rho}_{\mathrm{B}}=\operatorname{Tr}_{\mathrm{A}}(\hat{\rho})$; and calculate the von Neumann entropy $S=-\operatorname{Tr}_{\mathrm{B}}\left(\hat{\rho}_{\mathrm{B}} \log \hat{\rho}_{\mathrm{B}}\right)$ [36]. Here $\hat{\rho}$ is the density matrix of the total system. We consider two types of partitions: In partition 1 [Fig. 4(a)], A is the first trimer, and B is the rest. In partition 2 [Fig. 4(b)], $\mathrm{A}$ is the first site of each trimer, and B is the rest. The system is translationally invariant for each trimer. Thus, these two partitions highlight the local 

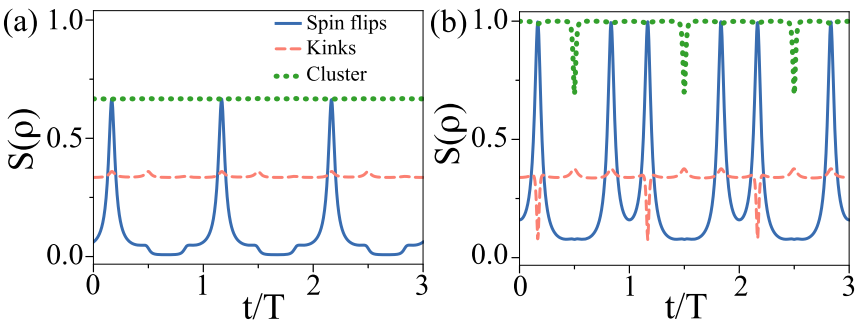

FIG. 4. Evolution of von Neumann entropy for the dual models with two types of partitions: (a) first trimer and (b) first site of each trimer that is traced out. The degree of entanglement as measured by the von Neumann entropy is normalized relative to the maximal possible entanglement. The parameters are the same as in Fig. 3.

and extended entanglement properties of the spins. We find that the entanglement exhibits dramatic changes during the pumping process. In particular, the entanglement of the spin flips increases at the anticrossings. Then, the spin flips are in a superposition state of being at two sites, which could be measured in experiments $[8,9]$. In contrast, the entangled kink and cluster states lose entanglement at the anticrossings for the case 2 partition, and have nearly unchanged entanglement for case 1 . The difference arises because partition 2 extends over all trimers, whereas partition 1 is measuring only a single trimer, highlighting the nonlocal structure of the quantum state. These properties can also be seen in the structure of the quantum states. The spin-flip, kink, and cluster-Ising Hamiltonians are related by duality transformation, which changes the wave function and entanglement profoundly. We can calculate the states for a single excitation per trimer exactly in the limit $g_{0} \gg J$ in Table I at and away from anticrossings. For our chosen values $\left(g_{0}=10 \mathrm{~J}, g_{1}=3 \mathrm{~J}\right)$, we find an overlap of the analytic states with the numeric results of more than $95 \%$ both at and away from the anticrossing. The overlap decreases with increasing $g_{0}$ or $J$ and is nearly unaffected by system size as we checked up to $N=9$.

\section{EFFECT OF DISORDER AND INTERACTIONS}

Topological pumping is generally considered with noninteracting particles. It is known that interactions can break topological pumping in some situations [28], while it is still possible in specific cases $[7,39]$. While some intuition about this question is present for the single-particle case, how does interaction affect pumping of nonlocal quantum correlations?

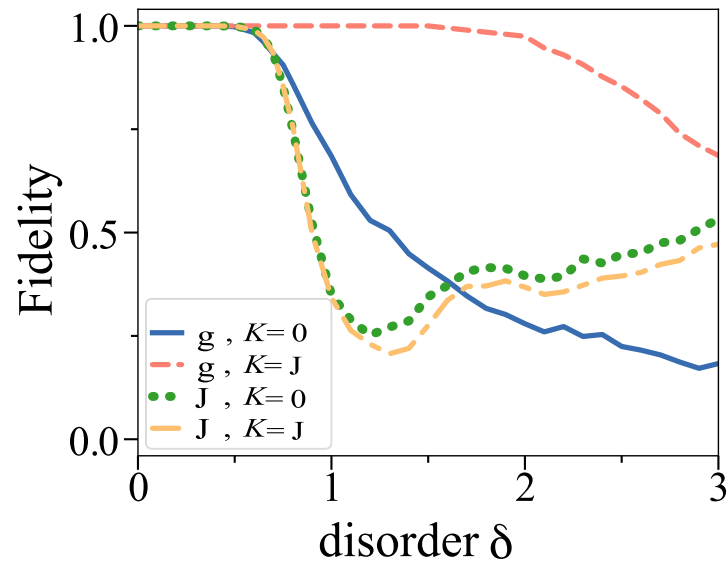

FIG. 5. Fidelity of the state $F=|\langle\Psi(0))| \Psi(3 T)\rangle\left.\right|^{2}$ after three pump cycles for random disorder strength $\delta$ applied either to $G_{j}(t)$ or to $J$ of the models. Disorder is sampled randomly between $[-\delta, \delta]$. Without interaction $K$, fidelity starts to decay when disorder is on the order of the energy gap $\delta_{c} \approx 0.7 \mathrm{~J}$, independent of which the variable is disordered. With interaction $K$, pumping is more robust for disorder in $G_{j}(t)$, however it is unchanged for $J$. Parameters are $N=9, g_{1}=3 J$, initial phase $\phi_{0}=0$, and angular frequency $\omega=0.02 J$.

Our scheme can shed light on this question, as the duality transformation can relate different types of interactions. We find that the lowest band with one excitation per trimer (Chern number $C=-1$ ) can be pumped even when adding the interaction term $\hat{H}_{\text {int }}^{\text {flip }}=\hbar K \sum_{j} \sigma_{j}^{x} \sigma_{j+1}^{x}$ to the spin-flip Hamiltonian $\hat{H}^{\text {flip }}$. However, interaction destroys pumping in the other bands. The pumping of that specific band persists as the single excitations of each trimer are highly localized and separated by a large distance of three sites. Thus, they couple only weakly to each other and the energy gap is nearly unchanged compared to the noninteracting case. This reasoning is valid for any model that can be reduced to spin flips via the transformation $\hat{U}$. The corresponding transformed interaction terms are $\hat{H}_{\mathrm{int}}^{\mathrm{kink}}=\hbar K \sum_{j} \sigma_{j}^{z} \sigma_{j+2}^{z}$ for kink Hamiltonian Eq. (5) and $\hat{H}_{\text {int }}^{\text {cluster }}=\hbar K \sum_{j} \sigma_{j}^{z} \sigma_{j+1}^{y} \sigma_{j+2}^{y} \sigma_{j+3}^{z}$ for clusterIsing Eq. (4). Interaction has a profound impact on the robustness to disorder. We implement random spatial disorder $\Delta_{j}$ with strength $\delta$ in either variable $G_{j}^{\prime}(t)=G_{j}(t)+\Delta_{j}$ or $J^{\prime}=J+\Delta_{j}$ of our Hamiltonians, where $\Delta_{j}$ is randomly sampled between $[-\delta, \delta]$. In Fig. 5 we show the fidelity

TABLE I. Eigenstates with one excitation per trimer in the pumping process of the spin-flip, kink, and cluster-Ising models. We show the analytic states at and away from the anticrossing in the limit $g_{0} \gg J$. States are given in terms of the eigenstates of the $z$ basis $|0\rangle$ and $|1\rangle$ for kinks and in the $x$ basis for spin-flip and cluster-Ising models. For the cluster-Ising model we define the ground state of the cluster model as $\prod_{i=1}^{N} c \mu_{i, i+1}^{z}|000\rangle_{x}$, with $c \mu_{i, i+1}^{z}$ being the control phase gate acting on site $i, i+1$.

\begin{tabular}{lcc}
\hline \hline Model & Away from anticrossing & At anticrossing \\
\hline Spin flip & $|010\rangle \otimes \cdots \otimes|010\rangle_{x}$ & $\frac{\sqrt{3}}{\sqrt{2 L}}\left[(|01\rangle-|10\rangle)|0\rangle \otimes \cdots \otimes(|01\rangle-|10\rangle)|0\rangle_{x}\right]$ \\
Kink & $\frac{1}{\sqrt{2}}\left(|100\rangle \otimes \cdots \otimes|100\rangle+|011\rangle \otimes \cdots \otimes|011\rangle_{z}\right)$ & $\frac{\sqrt{3}}{\sqrt{4 L}}\left[|1\rangle(|0\rangle-|1\rangle)|0\rangle \otimes \cdots \otimes|1\rangle(|0\rangle-|1\rangle)|0\rangle_{z}+\right.$ \\
Cluster Ising & $\prod_{j=0}^{N / 3} \mu_{3 j+2}^{z} \prod_{i=1}^{N} c \mu_{i, i+1}^{z}|000\rangle \otimes \cdots \otimes|000\rangle_{x}$ & $\prod_{j=0}^{N / 3} \frac{1}{\sqrt{2}}\left(\mu_{3 j+2}^{z}-\mu_{3 j+1}^{z}\right) \prod_{i=1}^{N} c \mu_{i, i+1}^{z}|000\rangle \otimes \cdots \otimes|000\rangle_{x}$ \\
\hline
\end{tabular}




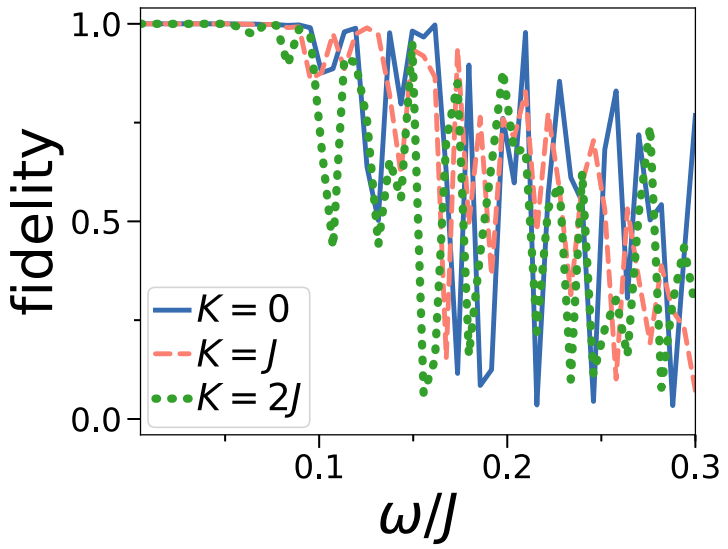

FIG. 6. Fidelity of the state $F=|\langle\Psi(0))| \Psi(9 T)\rangle\left.\right|^{2}$ after nine pump cycles for varying driving frequency $\omega$ and different interaction strength $K$. For $\omega<0.1 J$, pumping is stable. For higher frequencies, the fidelity starts to oscillate. Parameters are $N=9, g_{1}=3 J$, and initial phase $\phi_{0}=0$.

$F=|\langle\Psi(0) \mid \Psi(3 T)\rangle|^{2}$ of the pumped state after three pump cycles. We observe that without interaction $K=0$ the fidelity is reduced above a critical disorder, independent of whether disorder is applied to $G_{j}(t)$ or $J$. However, with interaction $K=J$, pumping is much more stable for disorder applied to $G_{j}(t)$. This effect cannot be attributed to a change in the energy gap, as it is nearly unchanged with interaction $K$. However, we observe that the system is more robust against disorder when the interaction operator commutes with the part of the Hamiltonian that is disordered, i.e., for the spin-flip model $\hat{H}_{\text {int }}^{\text {flip }}$ commutes with $\sigma_{i}^{x} \sigma_{i+1}^{x}$, but not with $\sigma_{i}^{z} \sigma_{i+1}^{z}$. We conjecture that the increased stability arises when the interaction Hamiltonian acts as a stabilizer on the pumped state, and leads to a renormalization of disorder due to interaction $g$ [40]. These findings on the stability against disorder found for spin flips can be immediately applied to pumping of kinks and the cluster-Ising model via the mapping scheme. In Fig. 6, we illustrate the frequency dependence of the pumping. We measure the overlap between the initial state and the pumped state after nine pumping cycles. Fidelity of the pumping is unity below a critical driving frequency. Interaction decreases the critical driving frequency slightly. Above this frequency, we observe strong oscillations in the pumping fidelity with frequency.

\section{CONCLUSIONS}

In conclusion, we have proposed a scheme to perform topological pumping of quantum correlations. Our approach is based on a unitary transformation that maps correlated states to uncorrelated ones, where known protocols for topological pumping can be used. To illustrate our general idea, we exploit spin dualities to realize topological pumping of cluster states, which are states relevant for quantum computation [29] or quantum memories [30]. We also show that the pumping process can reduce the bipartite entanglement for kinks and cluster excitations, but enhances it for single spin flips. Recent experiments have realized topological pumping of particles [8,9], without considering the entanglement. It would be of considerable interest to revisit these experiments to measure the entanglement dynamics. The spin-flip and kink models we considered are experimentally realizable in current quantum simulators [41-43] that are available in several platforms such as superconducting qubits [44-46], trapped ions [47], Rydberg atoms [48], and cold atoms [49-52]. Cluster states have been realized in photonic systems [53] and cluster Hamiltonians could be realized with cold atoms [54]. Our approach could be applied to find pumping schemes for other types of quantum correlations and to transport them, while being protected against disorder. Robustness against specific types of disorder can be enhanced by certain interaction terms, which can be determined by using our method. It would be interesting to investigate the implication of our results for error correction in spin chains [55] and the role of other type of dualities [56] on topological pumping.

\section{ACKNOWLEDGMENTS}

We thank D. G. Angelakis, M. Estarellas, M. Hanks, and H. Price for valuable discussions. We thank National Research Foundation Singapore, the Ministry of Education Singapore Academic Research Fund Tier 2 (Grant No. MOE2015-T21-101), and the Japanese QLEAP program for support. The Grenoble LANEF framework (ANR-10-LABX-51-01) is acknowledged for its support with mutualized infrastructure. The computational work for this paper was partially performed on resources of the National Supercomputing Centre, Singapore.

\section{APPENDIX A: HARPER-HOFSTADTER MODEL AND TOPOLOGICAL PUMPING IN THE AUBRY-ANDRE MODEL}

The Harper-Hofstadter model describes a Bloch electron moving in a two-dimensional lattice under the effect of a magnetic field:

$$
\begin{aligned}
\hat{H}_{\mathrm{HH}}= & -\hbar J_{x} \sum_{m_{x}, m_{y}}\left(f_{m_{x}, m_{y}}^{\dagger} f_{m_{x}+1, m_{y}}-f_{m_{x}, m_{y}} f_{m_{x}+1, m_{y}}^{\dagger}+\text { H.c }\right) \\
& -\hbar J_{y} \sum_{m_{x}, m_{y}}\left(e^{2 i \pi m_{x} b} f_{m_{x}, m_{y}}^{\dagger} f_{m_{x}, m_{y}+1}\right. \\
& \left.-e^{-2 i \pi m_{x} b} f_{m_{x}, m_{y}} f_{m_{x}, m_{y}+1}^{\dagger}\right) .
\end{aligned}
$$

Here $f_{m_{x}, m_{y}}$ and $f_{m_{x}, m_{y}}^{\dagger}$ are fermionic operators and $J_{x}$ and $J_{y}$ are hoppings strengths along $x$ and $y$ directions, respectively. When the electron completes a loop in the lattice, it acquires a phase $\phi$ proportional to the magnetic flux through the loop, as it is depicted in Fig. 7. Within a unit cell, the flux is denoted as $\phi=2 \pi b$, where $b$ is a real number. The physics of the Harper model is extremely rich and it is closely related to the integer quantum Hall effect and topological pumping. The reason for the nontrivial features of the model is the character of the real number $b$. For example, if it is a rational $b=p / q$ with $p, q$ integers, the magnetic flux through a unit cell is commensurable with the flux quanta. Figure 7(b) shows the case $b=1 / 3$, where the system is decomposed in terms of a sublattice. In this case, one can transform the Hamiltonian to 

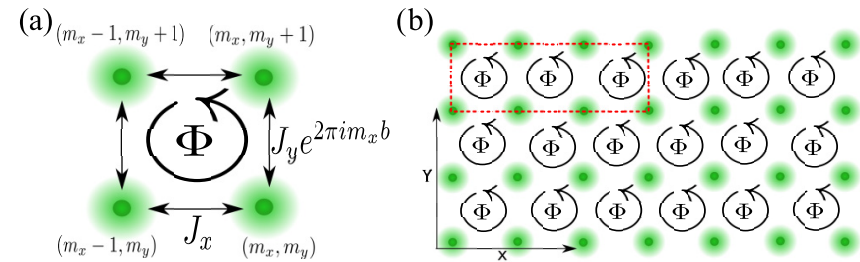

FIG. 7. The Harper-Hofstadter model of electrons in a twodimensional lattice. (a) Depiction of the flux through a unit cell. (b) The lattice described by the Harper-Hofstadter model.

quasimomentum representation, as follows:

$$
\hat{H}_{\mathrm{HH}}=\sum_{k_{x}, k_{y}} \Psi_{k_{x}, k_{y}}^{\dagger} \hat{H}_{k_{x}, k_{y}}^{\mathrm{HH}} \Psi_{k_{x}, k_{y}}^{\dagger},
$$

where $\Psi_{k_{x}, k_{y}}^{\dagger}=\left(F_{A, k_{x}, k_{y}}^{\dagger}, F_{B, k_{x}, k_{y}}^{\dagger}, F_{C, k_{x}, k_{y}}^{\dagger}\right)$. The operators $F_{n, k_{x}, k_{y}}^{\dagger}$ with $n \in\{A, B, C\}$ are the Fourier transform of the fermionic operators in real space. Due to our choice $b=1 / 3$, the Hamiltonian $\hat{H}_{k_{x}, k_{v}}^{\mathrm{HH}}$ is a $3 \times 3$ matrix and the system exhibits three bands that we denote by $\mathrm{A}, \mathrm{B}$, and $\mathrm{C}$, respectively. For a given band, $n \in\{A, B, C\}$, one can calculate the associated Chern number

$$
C_{n}=\frac{1}{2 \pi} \int_{\mathrm{FBZ}} \Omega_{n}\left(k_{x}, k_{y}\right) d k_{x} d k_{y},
$$

with the Berry curvature

$$
\begin{aligned}
\Omega_{n}\left(k_{x}, k_{y}\right)= & i\left(\left\langle\partial_{k_{x}} u_{n}\left(k_{x}, k_{y}\right) \mid \partial_{k_{y}} u_{n}\left(k_{x}, k_{y}\right)\right\rangle\right. \\
& -\left\langle\partial_{k_{y}} u_{n}\left(k_{x}, k_{y}\right)\left|\partial_{k_{x}} u_{n}(k, t)\right|\right),
\end{aligned}
$$

and $u_{n}\left(k_{x}, k_{y}\right)$ being the eigenstate of the $n$th band of the model in Fourier space.

An important step to establish the intimate relation between the Harper-Hofstadter model and topological pumping is to reduce the dimension of the system from a twodimensional lattice to a set of decoupled one-dimensional lattices. To achieve this, we assume periodic boundary conditions along the $y$ direction; one can transform the $y$ direction in Eq. (A1) into momentum representation, as follows:

$$
\begin{aligned}
\hat{H}_{\mathrm{HH}}= & -2 \hbar J_{y} \sum_{m_{x}, k_{y}} \cos \left(2 \pi b m_{x}+k_{y}\right)\left(f_{m_{x}, k_{y}}^{\dagger} f_{m_{x}, k_{y}}-f_{m_{x}, k_{y}} f_{m_{x}, k_{y}}^{\dagger}\right) \\
& -\hbar J_{x} \sum_{m_{x}, k_{y}}\left(f_{m_{x}, k_{y}}^{\dagger} f_{m_{x}+1, k_{y}}-f_{m_{x}, m_{y}} f_{m_{x}+1, k_{y}}^{\dagger}\right) \\
= & \sum_{k_{y}} \hat{H}\left(k_{y}\right) .
\end{aligned}
$$

This Hamiltonian $\hat{H}\left(k_{y}\right)$ is very important for our paper, because now we can modulate the quasimomentum $k_{y}=\omega t+$ $\phi_{0}$ adiabatically in time to perform topological pumping. After this procedure, we obtain the Aubry-Andre model:

$$
\begin{aligned}
\hat{H}_{\mathrm{AA}}(t)= & -2 \hbar J_{y} \sum_{j} \cos \left(2 \pi b j+\omega t+\phi_{0}\right)\left[f_{j}^{\dagger} f_{j}+\text { H.c }\right] \\
& -\hbar J_{x} \sum_{j}\left(f_{j}^{\dagger} f_{j+1}+\text { H.c }\right) .
\end{aligned}
$$

Note that as we are working with a one-dimensional system we consider an index $j$ to label the lattice sites. Similarly to the two-dimensional Harper-Hofstadter model Eq. (A1), for our choice $b=1 / 3$, the Hamiltonian Eq. (A6) exhibits three bands. This can be derived by Fourier transforming the annihilation and creation operators along the lattice index. Its bands have nontrivial topological Chern numbers, which are defined as

$$
C_{n}=\frac{1}{2 \pi} \int_{0}^{2 \pi} \int_{0}^{T} \Omega_{n}(k, t) d t d k,
$$

with the Berry curvature $\Omega_{n}(k, t)=i\left(\left\langle\partial_{t} u_{n}(k, t)\right|\right.$ $\left.\left.\partial_{k} u_{n}(k, t)\right\rangle-\left\langle\partial_{k} u_{n}(k, t) \mid \partial_{t} u_{n}(k, t)\right\rangle\right)$ and $u_{n}(k, t)$ being the eigenstate of the $n$th band of the model in Fourier space [8,33]. We neglected the band index $n$ for simplicity in the main text. The anomalous velocity $\dot{x}=\Omega_{n} \omega$ gives the speed with which the particles move during the pumping process. To understand the meaning of this quantity, let us consider an initial state $|\psi(0)\rangle$, representing a particle localized at a site $j_{0}$ along the lattice. Depending on the position $j_{0}$, the mean energy of the particle should be in one of the bands A, B, or C of the system. Now we can define the operator $\hat{x}=\sum_{j} j f_{j}^{\dagger} f_{j}$ in such a way that $x(t)=\langle\psi(t)|\hat{x}| \psi(t)\rangle$. After a single period $T$ of the time modulation, the position of the particle changes proportionally to the Chern number, as $x(T)-x(0)=C_{n}$, where $n$ is the band index and $x(0)$ is proportional to $j_{0}$ [57] (see also Fig. 3 in main text).

\section{APPENDIX B: MAPPING THE ISING MODEL TO THE AUBRY-ANDRE MODEL IN THE LIMIT OF WEAK SPIN INTERACTIONS}

The excitations above the paramagnetic ground state of the spin-flip Hamiltonian $\hat{H}^{\text {flip }}(t)$ in Eq. (3) of the main text are spin flips. In general, in the one-dimensional quantum Ising model the number of spin flips $\hat{\mathcal{N}}^{\text {flips }}=$ $1 / 2 \sum_{j=1}^{N}\left(1-\sigma_{j}^{x}\right)$ is not conserved. However, if the coupling $J$ between the spins in the $z$ direction is weak, $\hat{\mathcal{N}}^{\text {flips }}$ is approximately conserved, and we can obtain an effective Hamiltonian that preserves the number of spin flips, even under an adiabatic modulation of the onsite energies $G_{j}(t)=g_{0}+g_{1} \cos \left[2 \pi(j-1) b+\omega t+\phi_{0}\right]$. In order to do this, we go to a rotating frame with the unitary operator $\hat{V}(t)=\exp \left(-i g_{0} t \sum_{j=1}^{N} \sigma_{j}^{x}\right)$, where the Hamiltonian is given by $\hat{H}_{R}^{\text {flip }}(t)=\hat{V}^{\dagger}(t)\left(\hat{H}^{\text {flip }}(t)-i \hbar \partial_{t}\right) \hat{V}(t)$. After neglecting fast oscillating terms in $\hat{H}_{R}^{\text {flip }}(t)$ with frequencies proportional to $g_{0}$ and after going back to the laboratory frame, we obtain an effective Hamiltonian of the form

$$
\hat{H}^{\mathrm{flip}}(t) \approx \hbar \sum_{j=1}^{N}\left[-G_{j}(t) \sigma_{j}^{x}+J\left(\sigma_{j}^{z} \sigma_{j+1}^{z}+\sigma_{j}^{y} \sigma_{j+1}^{y}\right)\right],
$$

which is valid in the limit $g_{0} \gg J$. Under the rotating wave approximation, one just keeps terms in the Hamiltonian that preserve the number of spin flips. In this case, one can use the Jordan-Wigner transformation

$$
\begin{aligned}
& \sigma_{l}^{z}=-f_{l}^{\dagger} e^{i \hat{\Phi}_{l}}-f_{l} e^{-i \hat{\Phi}_{l}}, \quad \sigma_{l}^{y}=-i f_{l}^{\dagger} e^{i \hat{\Phi}_{l}}+i f_{l} e^{-i \hat{\Phi}_{l}}, \\
& \sigma_{l}^{x}=2 f_{l}^{\dagger} f_{l}-1,
\end{aligned}
$$


with $\hat{\Phi}_{l}=\sum_{j<l} f_{j}^{\dagger} f_{j}$ to map Eq. (B1) to the Aubry-Andre model discussed above:

$$
\hat{H}^{\text {flip }}(t) \approx 2 \hbar \sum_{j=1}^{N}\left[-G_{j}(t) f_{j}^{\dagger} f_{j}+J\left(f_{j}^{\dagger} f_{j+1}+\text { H.c. }\right)\right],
$$

where $f_{j}^{\dagger}\left(f_{j}\right)$ are fermionic creation (annihilation) operators. With the definition of $G_{j}(t)$, this model maps to the AubryAndre model of Eq. (A6), which has been widely studied for topological pumping.

In the previous section, we discussed the relation between the Chern number and the change in the mean position $x$ of the particle. As we are working with spin flips here, we can define a single spin flip initially localized at a given site $j_{0}$. The position operator $\hat{x}=\sum_{j} j f_{j}^{\dagger} f_{j}$ of the spin flip is given by $\hat{x}=1 / 2 \sum_{j=1}^{N} j\left(\sigma_{j}^{x}+1\right)$ and its mean value reads $x(t)=\langle\psi(t)|\hat{x}| \psi(t)\rangle$, where $|\psi(t)\rangle$ represent the state during the pumping process. During topological pumping, the change of the position is related to the Chern number. In our case, we obtain the relation $x(T)-x(0)=1 / 2 \sum_{j=1}^{N} j\left(\left\langle\sigma_{j}^{x}(T)\right\rangle-\right.$ $\left.\left\langle\sigma_{j}^{x}(0)\right\rangle\right)=C_{n}$, where $C_{n}$ is the Chern number. Now if we take into account the duality, we can show that a similar relation is satisfied for clusterlike states:

$$
\begin{aligned}
x(T)-x(0)= & \frac{1}{2} \sum_{j=1}^{N} j\left(\left\langle\mu_{j}^{z} \mu_{j+1}^{x} \mu_{j+2}^{z}(T)\right\rangle\right. \\
& \left.-\left\langle\mu_{j}^{z} \mu_{j+1}^{x} \mu_{j+2}^{z}(0)\right\rangle\right)=C_{n} .
\end{aligned}
$$

In this case, however, the center of mass of the excitation, i.e., its mean position, is given by a three-point correlation function. A similar expression can be obtained for kinks, where the center of mass is obtained by calculating a twopoint correlation function.

\section{APPENDIX C: HIGHER-ORDER DUALITIES}

Our procedure can also reduce more complex models to single particles. For example, let us consider the many-body operator

$$
\hat{O}(t)=-\hbar(-1)^{r} \sum_{j=1}^{N} G_{j}(t) \tilde{\mu}_{j-1}^{z}\left[\prod_{m=0}^{r} \tilde{\mu}_{m+j}^{x}\right] \tilde{\mu}_{j+1+r}^{z},
$$

where $r$ is a positive integer. By repeatedly applying $\pi$ rotations around the $x$ axis of the Pauli matrices and then applying the duality, it can be subsequently reduced to spin flips $\hat{O}_{\text {single }}(t)=-\hbar \sum_{j} G_{j}(t) \sigma_{j}^{x}$. After this reduction, it is quite simple to find the operator $\hat{B}_{\text {single }}=\hbar J \sum_{j} \sigma_{j}^{z} \sigma_{j+1}^{z}$ that allows us to perform topological pumping of spin flips. Now we can apply the inverse transformation and obtain the operator $\hat{B}=\hbar(-1)^{r} J \sum_{j=1}^{N} \tilde{\mu}_{j-1}^{z}\left[\prod_{m=0}^{r-1} \tilde{\mu}_{m+j}^{x}\right] \tilde{\mu}_{j+r}^{z}$. By considering the aforementioned operators, we can construct the Hamiltonian

$$
\begin{aligned}
\hat{H}(t)= & -\hbar(-1)^{r} \sum_{j=1}^{N} G_{j}(t) \tilde{\mu}_{j-1}^{z}\left[\prod_{m=0}^{r} \tilde{\mu}_{m+j}^{x}\right] \tilde{\mu}_{j+1+r}^{z} \\
& +\hbar(-1)^{r} J \sum_{j=1}^{N} \tilde{\mu}_{j-1}^{z}\left[\prod_{m=0}^{r-1} \tilde{\mu}_{m+j}^{x}\right] \tilde{\mu}_{j+r}^{z},
\end{aligned}
$$

which allows us to pump highly correlated states. As a direct consequence, the $(r+2)$-point correlation function $\Delta_{j-1, j, \ldots, j+1+r}(t)=\left\langle\tilde{\mu}_{j-1}^{z}\left[\prod_{m=0}^{r} \tilde{\mu}_{m+j}^{x}\right] \tilde{\mu}_{j+1+r}^{z}(t)\right\rangle$ satisfies

$$
\begin{aligned}
x(T)-x(0)= & \frac{1}{2} \sum_{j=1}^{N} j\left[\Delta_{j-1, j, \ldots, j+1+r}(T)\right. \\
& \left.-\Delta_{j-1, j, \ldots, j+1+r}(0)\right]=C_{n} .
\end{aligned}
$$

This equation establishes a link between quantum correlations and the Chern number, which is a topological quantity.
[1] D. J. Thouless, Phys. Rev. B 27, 6083 (1983).

[2] Q. Niu and D. J. Thouless, J. Phys. A 17, 2453 (1984).

[3] J. E. Avron and R. Seiler, Phys. Rev. Lett. 54, 259 (1985).

[4] F. Mei, J.-B. You, D.-W. Zhang, X. C. Yang, R. Fazio, S.-L. Zhu, and L. C. Kwek, Phys. Rev. A 90, 063638 (2014).

[5] L. Zhou, D. Y. Tan, and J. Gong, Phys. Rev. B 92, 245409 (2015).

[6] R. W. Bomantara, G. N. Raghava, L. Zhou, and J. Gong, Phys. Rev. E 93, 022209 (2016).

[7] J. Tangpanitanon, V. M. Bastidas, S. Al-Assam, P. Roushan, D. Jaksch, and D. G. Angelakis, Phys. Rev. Lett. 117, 213603 (2016).

[8] M. Lohse, C. Schweizer, O. Zilberberg, M. Aidelsburger, and I. Bloch, Nat. Phys. 12, 350 (2016).

[9] S. Nakajima, T. Tomita, S. Taie, T. Ichinose, H. Ozawa, L. Wang, M. Troyer, and Y. Takahashi, Nat. Phys. 12, 296 (2016).

[10] Y. E. Kraus, Y. Lahini, Z. Ringel, M. Verbin, and O. Zilberberg, Phys. Rev. Lett. 109, 106402 (2012).
[11] M. Verbin, O. Zilberberg, Y. Lahini, Y. E. Kraus, and Y. Silberberg, Phys. Rev. B 91, 064201 (2015).

[12] T. Kitagawa, E. Berg, M. Rudner, and E. Demler, Phys. Rev. B 82, 235114 (2010).

[13] M. Lohse, C. Schweizer, H. M. Price, O. Zilberberg, and I. Bloch, Nature (London) 553, 55 (2018).

[14] O. Zilberberg, S. Huang, J. Guglielmon, M. Wang, K. P. Chen, Y. E. Kraus, and M. C. Rechtsman, Nature (London) 553, 59 (2018).

[15] H. A. Kramers and G. H. Wannier, Phys. Rev. 60, 252 (1941).

[16] H. A. Kramers and G. H. Wannier, Phys. Rev. 60, 263 (1941).

[17] J. B. Kogut, Rev. Mod. Phys. 51, 659 (1979).

[18] R. Savit, Rev. Mod. Phys. 52, 453 (1980).

[19] A. Strominger, S.-T. Yau, and E. Zaslow, Nucl. Phys. B 479, 243 (1996).

[20] E. Cobanera, G. Ortiz, and Z. Nussinov, Adv. Phys. 60, 679 (2011). 
[21] N. Seiberg, T. Senthil, C. Wang, and E. Witten, Ann. Phys. (NY) 374, 395 (2016).

[22] P. Sacramento and V. Vieira, Ann. Phys. (NY) 391, 216 (2018).

[23] J. Murugan and H. Nastase, J. High Energy Phys. 05 (2017) 159.

[24] D. F. Mross, J. Alicea, and O. I. Motrunich, Phys. Rev. X 7, 041016 (2017).

[25] W. Son, L. Amico, F. Plastina, and V. Vedral, Phys. Rev. A 79, 022302 (2009).

[26] S. Sachdev, Quantum Phase Transitions, 2nd ed. (Cambridge University Press, Cambridge, England, 2011).

[27] W. Son, L. Amico, R. Fazio, A. Hamma, S. Pascazio, and V. Vedral, Europhys. Lett. 95, 50001 (2011).

[28] M. Nakagawa, T. Yoshida, R. Peters, and N. Kawakami, Phys. Rev. B 98, 115147 (2018).

[29] R. Raussendorf, D. E. Browne, and H. J. Briegel, Phys. Rev. A 68, 022312 (2003).

[30] M. Goihl, N. Walk, J. Eisert, and N. Tarantino, arXiv:1908.10869.

[31] S. Aubry and G. André, Ann. Israel Phys. Soc 3, 18 (1980).

[32] D. R. Hofstadter, Phys. Rev. B 14, 2239 (1976).

[33] D. Xiao, M.-C. Chang, and Q. Niu, Rev. Mod. Phys. 82, 1959 (2010).

[34] S. O. Skrøvseth and S. D. Bartlett, Phys. Rev. A 80, 022316 (2009).

[35] A. C. Doherty and S. D. Bartlett, Phys. Rev. Lett. 103, 020506 (2009).

[36] P. Smacchia, L. Amico, P. Facchi, R. Fazio, G. Florio, S. Pascazio, and V. Vedral, Phys. Rev. A 84, 022304 (2011).

[37] W. Son, L. Amico, and V. Vedral, Quantum Inf. Process. 11, 1961 (2012).

[38] W. Nie, F. Mei, L. Amico, and L. C. Kwek, Phys. Rev. E 96, 020106(R) (2017).

[39] T. Haug, R. Dumke, L.-C. Kwek, and L. Amico, Commun. Phys. 2, 127 (2019).

[40] D. Yue, L. I. Glazman, and K. A. Matveev, Phys. Rev. B 49, 1966 (1994).

[41] I. M. Georgescu, S. Ashhab, and F. Nori, Rev. Mod. Phys. 86, 153 (2014).
[42] C. Noh and D. G. Angelakis, Rep. Prog. Phys. 80, 016401 (2017).

[43] M. J. Hartmann, J. Opt. 18, 104005 (2016).

[44] P. Roushan, C. Neill, J. Tangpanitanon, V. M. Bastidas, A. Megrant, R. Barends, Y. Chen, Z. Chen, B. Chiaro, A. Dunsworth et al., Science 358, 1175 (2017).

[45] C. Neill, P. Roushan, K. Kechedzhi, S. Boixo, S. V. Isakov, V. Smelyanskiy, A. Megrant, B. Chiaro, A. Dunsworth, and K. Arya, Science 360, 195 (2018).

[46] K. Xu, J.-J. Chen, Y. Zeng, Y.-R. Zhang, C. Song, W. Liu, Q. Guo, P. Zhang, D. Xu, H. Deng et al., Phys. Rev. Lett. 120, 050507 (2018).

[47] J. Smith, A. Lee, P. Richerme, B. Neyenhuis, P. W. Hess, P. Hauke, M. Heyl, D. A. Huse, and C. Monroe, Nat. Phys. 12, 907 (2016).

[48] H. Weimer, M. Müller, H. P. Büchler, and I. Lesanovsky, Quantum Inf. Process. 10, 885 (2011).

[49] M. Schreiber, S. S. Hodgman, P. Bordia, H. P. Lüschen, M. H. Fischer, R. Vosk, E. Altman, U. Schneider, and I. Bloch, Science 349, 842 (2015).

[50] J.-Y. Choi, S. Hild, J. Zeiher, P. Schauß, A. Rubio-Abadal, T. Yefsah, V. Khemani, D. A. Huse, I. Bloch, and C. Gross, Science 352, 1547 (2016).

[51] L. Amico, G. Birkl, M. Boshier, and L.-C. Kwek, New J. Phys. 19, 020201 (2017).

[52] R. Dumke, Z. Lu, J. Close, N. Robins, A. Weis, M. Mukherjee, G. Birkl, C. Hufnagel, L. Amico, M. G. Boshier et al., J. Opt. 18, 093001 (2016).

[53] K. Chen, C.-M. Li, Q. Zhang, Y.-A. Chen, A. Goebel, S. Chen, A. Mair, and J.-W. Pan, Phys. Rev. Lett. 99, 120503 (2007).

[54] J. K. Pachos and M. B. Plenio, Phys. Rev. Lett. 93, 056402 (2004).

[55] F. G. S. L. Brandão, E. Crosson, M. B. Sahinoglu, and J. Bowen, Phys. Rev. Lett. 123, 110502 (2019).

[56] A. Almheiri, X. Dong, and D. Harlow, J. High Energy Phys. 04 (2015) 163.

[57] M. Aidelsburger, M. Lohse, C. Schweizer, M. Atala, J. T. Barreiro, S. Nascimbene, N. Cooper, I. Bloch, and N. Goldman, Nat. Phys. 11, 162 (2015). 